\title{
IMAGING OF LYMPHATIC DYSPLASIA IN NOONAN SYNDROMIE: CASE STUDIES AND HISTORICAL ATLAS
}

\author{
T. Cox, C. Vance, S. Daley, C. Papendieck, H. McGregor, P.H. Kuo, M.H. Witte
}

Department of Surgery (TC,CV,SD,MHW) and Medical Imaging (HM,PHK), University of Arizona, Tucson, Arizona USA; Angiopediatria (CP), Buenos Aires, Argentina

\section{ABSTRACT}

To determine the historical use and utility of various lymphatic imaging modalities in Noonan syndrome (NS) patients, we performed a comprehensive literature review by collecting the published medical imaging of NS lymphatic dysplasias. We correlated imaging findings with clinical phenotypes and treatment. Our analysis of lymphatic imaging modalities provides an algorithmic approach to imaging and patient care across the spectrum of NS developmental defects. A total of 54 NS cases have been published since 1975. Using the observations reported in 15 reviewed publications, an association was made between disruptions in central lymphatic flow and poor clinical presentations/outcomes in NS patients.

Keywords: Noonan syndrome, lymphatic vessel dysplasia, lymphatic imaging, lymphangiography, thoracic duct, imaging, algorithm, atlas

Noonan syndrome (NS) is a congenital disorder that arises from mutations in several previously identified genes which result in overactivation of the RAS/RAF/ERK signaling pathways (1). These mutations most often arise spontaneously but may also be inherited in an autosomal dominant pattern and is estimated to occur with a frequency of 1 in 1000-2500 live births, with equal prevalence in both sexes and across all ethnicities (1).

Patients with a suspected diagnosis of NS classically display a pleiomorphic combination of features such as short stature, webbed neck, cystic hygroma, skeletal abnormalities, congenital heart defects (CHDs), especially pulmonary stenosis, lymphedema, facial dysmorphism, and cognitive deficits (1). These features are similar to those found in Turner syndrome (TS), a phenotypically similar but genetically distinct disorder that is only present in females with an XO sexchromosome monosomy. Clinical descriptions of a "male" TS existed prior to the 1960's. However, it was not until 1962 when Jacqueline Noonan, a pediatric cardiologist, initially presented her findings of a "male Turner syndrome" phenotype (2) that described a novel, distinct clinical entity found equally in both males and females. Her findings were published in 1968, and the condition would later be named Noonan syndrome (2).

Lymphatic involvement in NS pathogennesis did not begin to receive attention until 1974 after the publication of a case series by Minkin et al (3), which characterized and 
analyzed the congenital lymphatic abnormalities observed in many patients with NS. Since that time, a few studies have collectively found that congenitally acquired lymphatic dysfunction or dysplasia is an etiological hallmark of NS pathogenesis and phenotypic variability (4-6). Although most NS studies have described various operative and nonoperative methods to manage the lymphatic sequelae associated with NS, only a limited number of studies exist which specifically address the usefulness of lymphatic imaging to aid in the characterization of NS disease manifestations. Essentially every form of current medical imaging (X-ray, CT, MRI, nuclear medicine, etc.) has practical applications in visualizing the lymphatic system and are featured in this review.

We propose that early and regular lymphatic imaging should be highly prioritized by physicians to diagnose lymphatic abnormalities and help guide the medical and/ or surgical treatment of patients with NS. Our historically comprehensive evaluation of the various available lymphatic imaging modalities supports a central role for lymphatic imaging in both operative and nonoperative management of the lymphatic sequelae associated with NS.

\section{METHODS}

\section{Literature Search}

We used the PubMed database to find all the studies included in this literature review. We searched "Noonan AND Lymphatic AND Imaging" which yielded 36 results. Database searches covered all time periods. Inclusion criteria for this study were: 1) diagnosis and clinical description of patients with NS, and 2) observations contained within the publication showcased imaging studies and/or results analysis of imaged lymphatic structure, function, or sequelae. We accepted studies which described imaging results without necessarily including images. Documentation of surgical interventions or genetic testing were not criteria for inclusion. Of the 36 original PubMed papers, 25 met our inclusion criteria.

We excluded publications featuring ultrasound (US) diagnosis of NS in utero since available research suggests fetal US findings do not correlate with post-natal patient outcomes or potential disease severity (7). We also excluded clinical descriptions of other medical conditions or similar genetic syndromes unless comorbid NS diagnosis could be confirmed. Of the 25 papers that met our inclusion criteria, 8 were excluded based on these exclusion criteria.

Using the inclusion and exclusion criteria mentioned above, 17 publications from our PubMed search were eligible for inclusion in our review. By individually searching through the references cited by each of these selected publications, 4 more qualifying papers were added for a total of 21 publications with 52 patients. Reviewed articles were listed in chronological order since 1975 and designated as "study" \#1-21(See below "Literature Review Articles - Chronologic"). Clinical descriptions and imaging results from 3 of our own patients with NS were also included, and these cases are described below in Case Presentations. One of our 3 patients was not counted in the patient total since his pre-operative lymphatic imaging and preliminary surgical results were published previously by Howarth et al (8) and was previously counted in our review of cases. Thus, our total patient count was 54. Lymphatic imaging results and clinical symptoms were compared between our patients and those from the reviewed literature.

\section{Imaging Techniques}

Before introducing the specific case presentations, it is essential to understand the imaging techniques used to identify lymphatic abnormalities. There are three main types of imaging techniques used to visualize the lymphatic system including conventional oil 
contrast lymphangiography, magnetic resonance lymphography, and lymphangioscintigraphy. We describe these techniques in further detail and how they were employed in the workup of our patients with Noonan syndrome. The pros and cons of these techniques are described in the Discussion section.

\section{Conventional oil-lymphangiography}

Conventional oil-lymphangiography (CLAG) and CT lymphography (CTL) is performed by injecting a lipid-based radiocontrast dye (iodized lipidiol) directly into lymph nodes or via cannulation of peripheral lymphatic vessels, followed by visualization on $\mathrm{X}$ ray or CT, respectively. Specialized fluorescence-based lymphangiography utilizes indocyanine green (I-LAG).

\section{Magnetic resonance lymphography}

Magnetic resonance lymphography (MRL) visualizes the lymphatic system using a T2-weighted MRI with modified parameters, which highlights lymphatic fluid without the use of a contrast agent. Dynamic-contrast MR lymphography (dc-MRL) first utilizes USguided intranodal injection of a gadoliniumbased contrast agent. Then, MRI dynamically captures modified T1-weighted images over specified time intervals, illustrating the lymphatic vasculature of the patient while identifying fluid leaks into other tissues as they appear.

\section{Lymphangioscintigraphy (LAS)}

Lymphangioscintigraphy (LAS) is performed by intradermal injection of a radiolabeled tracer (i.e., technetium- $99 \mathrm{~m}$ labeled sulfur colloid or albumin) into the patient's feet and/or hands. The tracer is then taken up by the lymphatic system and images are acquired at various intervals over the course of several hours. Imaging is performed using standard gamma cameras, which detect internal radiation in a variety of imaging contexts within the field of nuclear medicine. In planar imaging, the camera's detector heads do not rotate, and therefore collect twodimensional images only (9). In contrast, with single photon emission computed tomography (SPECT) detector heads rotate during the image capture process and therefore acquire a three-dimensional image of the distribution of the radiotracer (9). SPECT and CT can be simultaneously collected with a hybrid SPECT/CT scanner, and a 3-D image of the SPECT fused together onto the CT is constructed.

\section{CASE PRESENTATIONS}

\section{Patient 1}

Patient 1 (P1) was a 35-year-old male with NS phenotype, cryptorchidism (corrected at age 11), and hemodynamically stable congenital heart defects, including pulmonic stenosis, aortic aneurysm, mitral and tricuspid insufficiency. At age 15, the patient noticed scrotal blisters leaking cloudy pink fluid along with occasional lymphedema of the feet and ankles bilaterally. At age 23 he was evaluated using C-LAG and LAS which revealed severe retroperitoneal lymphangiectasia and chylous reflux into the perineum, scrotum, and penis. Lympho-venous shunt surgery was performed, and imaging findings were published by Howarth et al (8). At age 35 he presented to us for assessment following chronic post-operative recurrence of scrotal edema, clear-yellow leakage of blistering scrotal lesions, and occasional foot and ankle swelling. LAS, MRL, and CT imaging was performed and compared to published pre-operative images 12 years prior.

P1's lower extremity LAS effectively visualized the deep lymphatic trunks and regional lymph nodes bilaterally. Following right foot injection of radiotracer (1.5 hours prior to left foot injection), there was no reflux seen in the scrotum. However, prompt reflux was seen from the groin lymphatics into the scrotum on the left. Scattered lymph nodes 


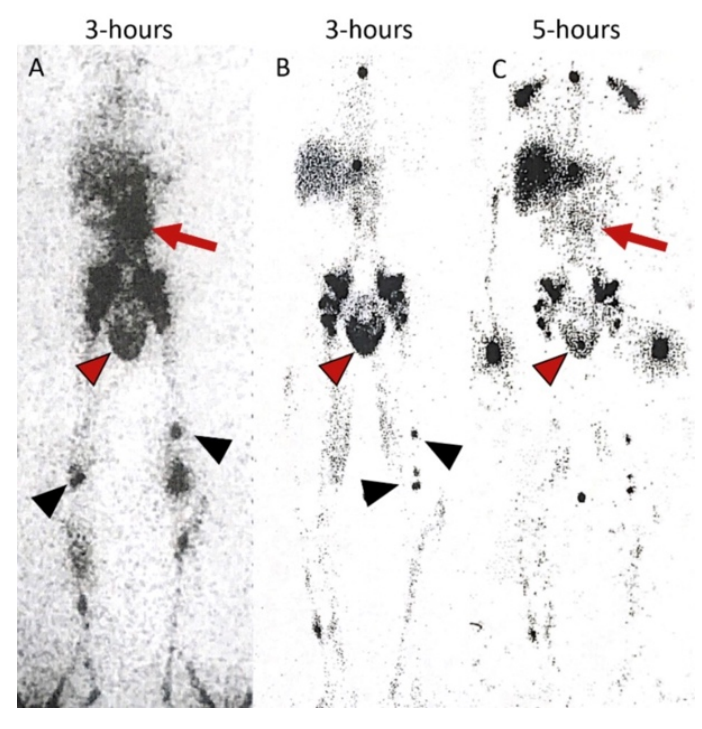

Fig. 1: Pre- and Post-Op anterior view LAS imaging for Patient 1. 1A: Pre-Operative anterior view LAS; age 23. 1B: Post-Operative anterior view LAS; age 35. 1C: Post-Operative anterior view LAS; 5-hour delay, age 35. Red arrow: Abdominal diffusion improved post-op. Red arrowhead: Scrotal reflux improved post-op. Black arrowhead: Dermal reflux, which indicates valvular incompetence secondary to obstruction.

nodes and tracer accumulation were seen centrally and diffusely in the abdomen/upper retroperitoneum with suggestive visualization of the thoracic duct on the delayed film. Popliteal and other lymph accumulations along the lower extremities were diminished compared to pre-operative LAS (Fig. 1). CT revealed dilated and tortuous lymphatic vessels of the abdomen consistent with the patient's previously published C-LAG imaging. MRL imaging of the abdomen and pelvis showed interval increase in scrotal edema along with a small area of previously unobserved lymph collection in the obturator region of the left inferior pelvis (Fig. 2).

\section{Patient 2}

Patient 2 (P2) was an 11-year-old female who exhibited a complex disease presentation including craniofacial and musculoskeletal abnormalities, intellectual disability, pulmonic valve stenosis (corrected by balloon angioplasty), mitral valve regurgitation, pulmonary lymphangiectasia, recurrent episodes of upper-respiratory infection/pneumonia, bilateral lymphedema of the feet, and diffuse giant-cell granulomas of the maxilla/mandible, sinuses, clavicle, wrist, knees, and ankles. Diagnosis of NS was confirmed by identification of a de novo mutation in the SOS1 gene of the RAS/RAF/ERK signaling pathway, a mutation seen in some patients with NS (10). At the age of eight she presented with inguinal lymphadenopathy and bilateral swelling of the lower legs and underwent LAS imaging. When she later presented with pulmonary lymphangiectasias and frequent dry cough, a comprehensive evaluation including genetic testing, and lymphatic imaging were undertaken to address her progressively deteriorating respiratory status and cardiac function, as well as worsening hepatosplenomegaly, severe malnutrition/cachexia, and unexplained anemia. Whole body LAS imaging was performed with SPECT/CT. To our knowledge, this method for evaluating lymphatics has not been previously reported in patients with NS. T2weighted MRL was also performed to confirm overall SPECT/CT structural findings.

On CT imaging, a tortuous mass of vessels was identified in the upper mediastinum, which extended into the neck and appeared to impinge on the upper trachea. MR imaging confirmed these findings. Corresponding areas of radiotracer uptake on SPECT/CT were seen accumulating in the right hilum and lung field. Tracer accumulation was also observed in the abdomen, and only the lower portion of the thoracic duct was visible (Fig. 3-5).

\section{Patient 3}

Patient 3 (P3) was a 36-year-old male with cryptorchidism corrected at birth and a clinical diagnosis of NS since age 8. At age 10, he presented with onset of asymmetric 

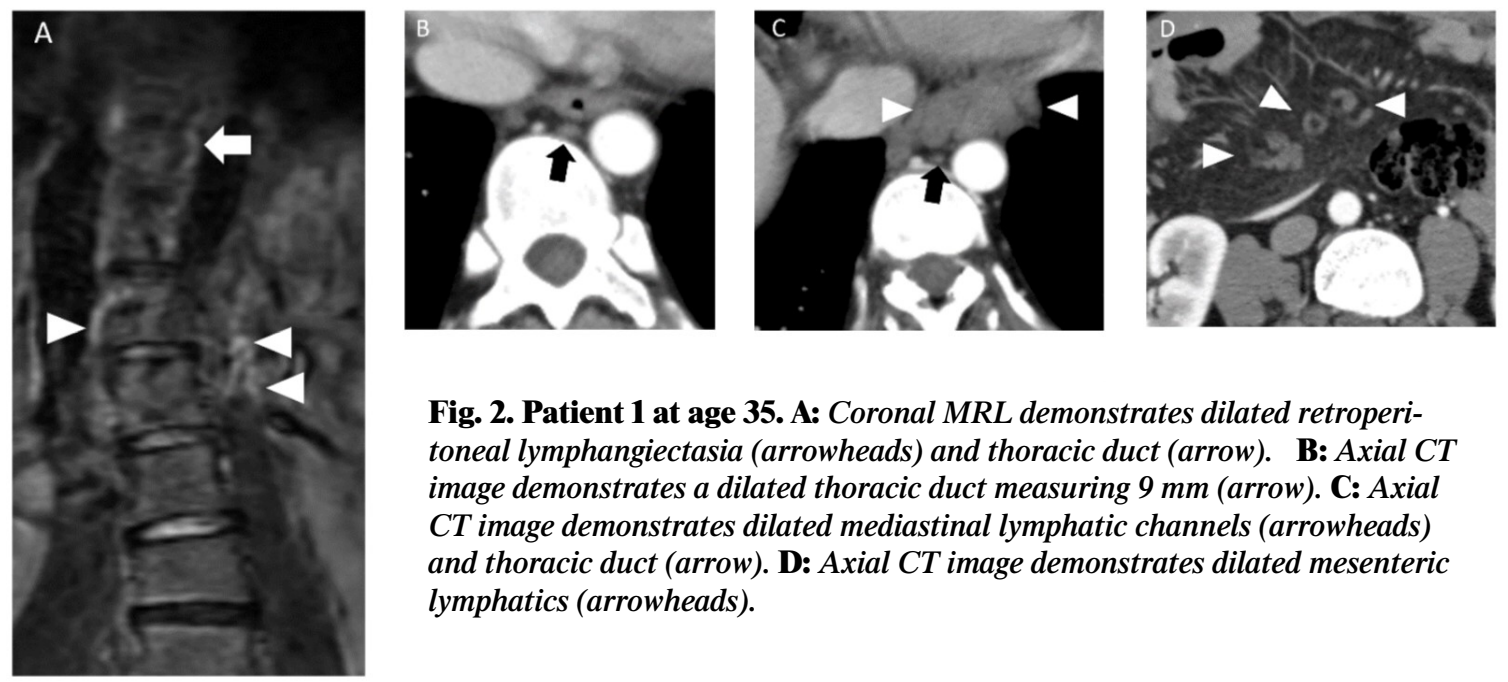

Fig. 2. Patient 1 at age 35. A: Coronal MRL demonstrates dilated retroperitoneal lymphangiectasia (arrowheads) and thoracic duct (arrow). B: Axial CT image demonstrates a dilated thoracic duct measuring $9 \mathrm{~mm}$ (arrow). C: Axial CT image demonstrates dilated mediastinal lymphatic channels (arrowheads) and thoracic duct (arrow). D: Axial CT image demonstrates dilated mesenteric lymphatics (arrowheads).

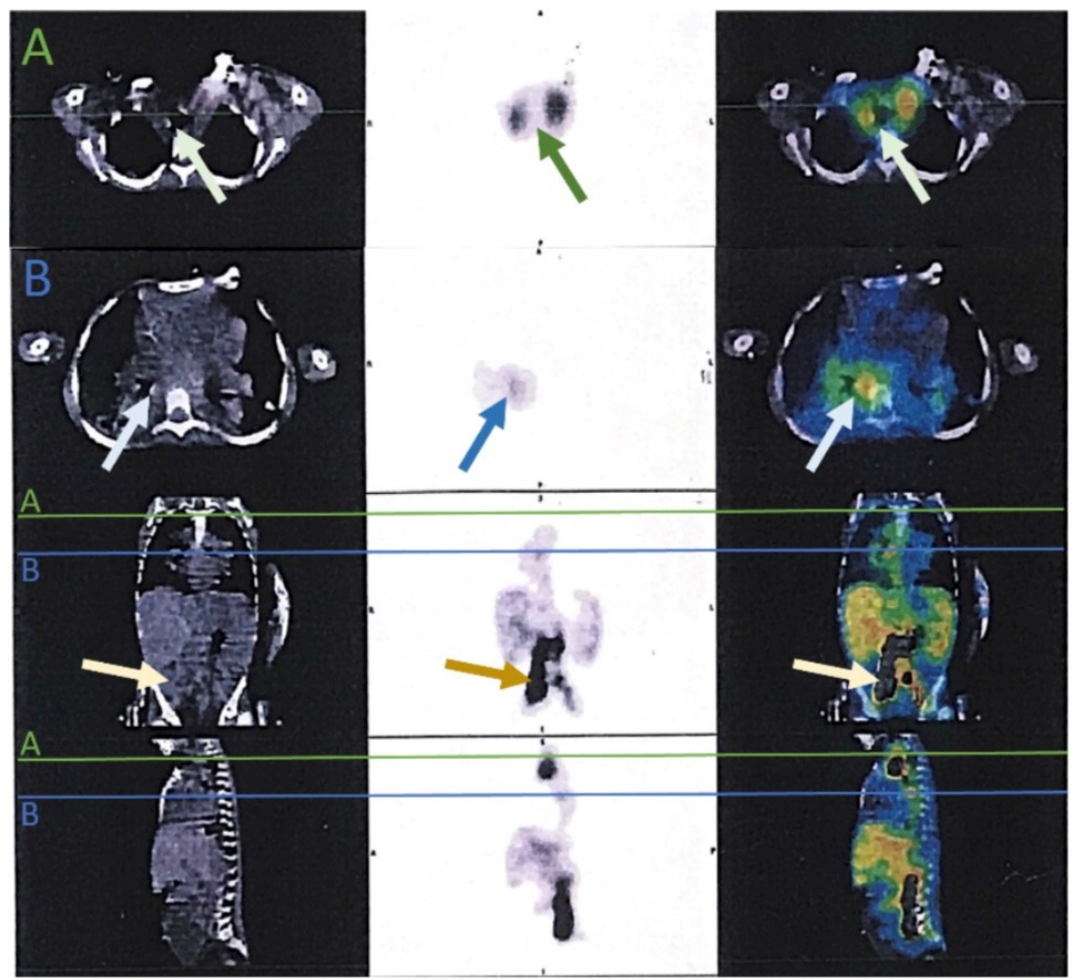

Fig. 3. SPECT/CT of Patient 2 at age eleven. Left-CT only. Middle-SPECT only. Right-fused SPECT/CT images. A tortuous mass is visible in the upper mediastinum which extends from the right hilum up to the neck and impinges on the trachea. Radiotracer accumulations confirm lymphatic etiology. Color coded lines $\mathbf{A}$ and $\mathbf{B}$ on coronal and sagittal plane images (bottom two rows) correspond to axial-plane images (top two rows). Impingement of the upper/lower respiratory tract (green and blue arrows) and the presence of dilated inguinal lymphatics (yellow arrows) are evident. 


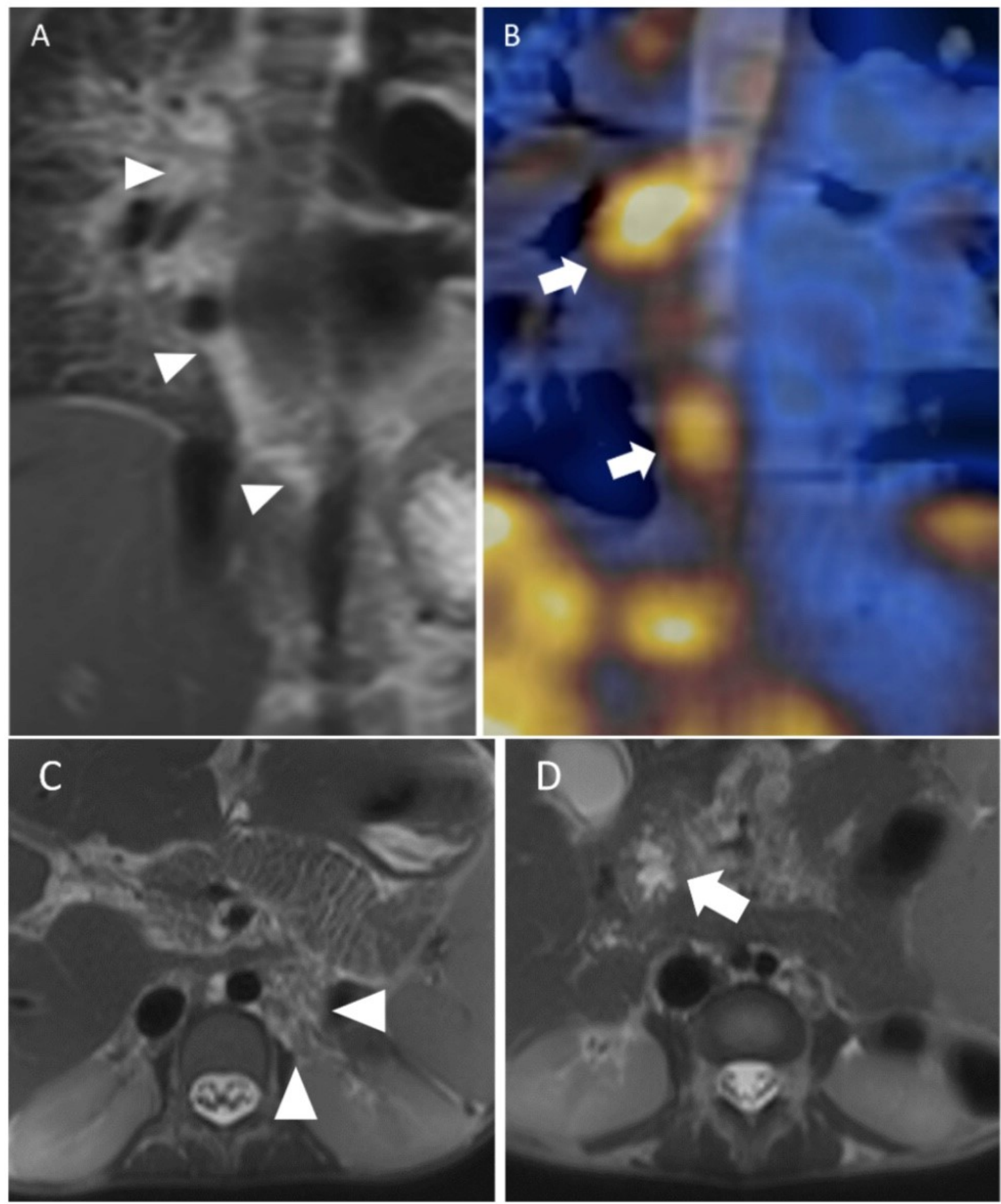

Fig. 4. Patient 2. A: Coronal MRL demonstrates dilated and tortuous lymphatic vessels extending along the right aspect of the mediastinum into the right hilum (arrowheads). B: Corresponding radiotracer accumulation from coronal SPECT/CT imaging (arrows). C and D: Axial MRL images demonstrate prominent retroperitoneal (arrowheads) and duodenal (arrow) lymphatics. 

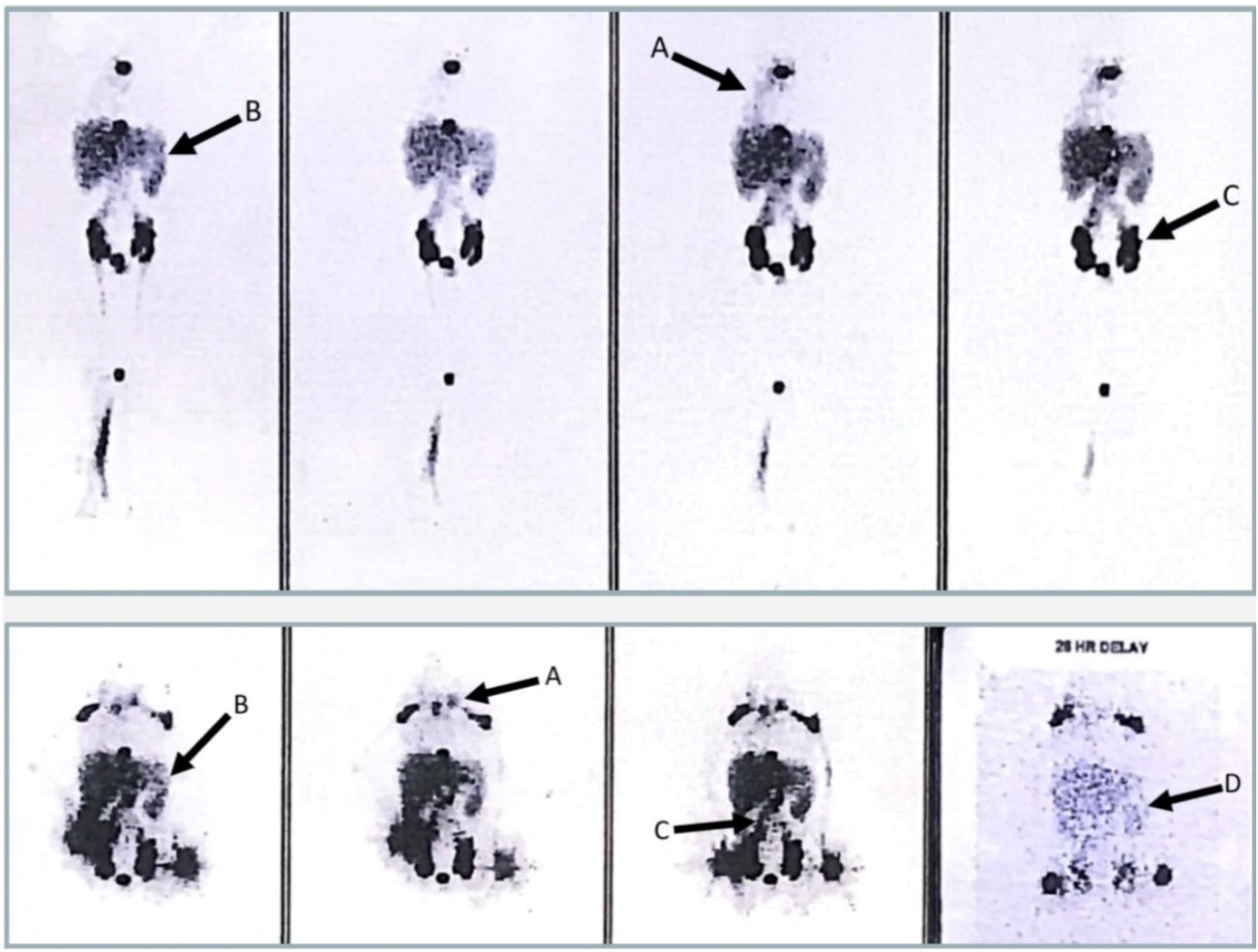

Fig. 5. LAS imaging of Patient 2 at age 11. Mediastinal/pulmonary reflux visualized (arrow A), prominent uptake in the enlarged spleen (arrow B), and dilated inguinal lymphatic channels (arrow $C$ ). All of these features persist even after a 26-hour delay (arrow D).

asymmetric grade-II primary lymphedema of the lower extremities and a left malleolar lympho-cutaneous fistula, which prompted an LAS imaging study and lympho-venous shunt surgery. Chest X-ray (CXR) was obtained at age 36 to evaluate an acute chylothorax that was treated previously.

LAS revealed retroperitoneal and mediastinal lymphatic malformation with right deviation of the thoracic duct. CXR exhibited a fine reticulo-nodular pattern, abnormal retrosternal opacity, and chylous blunting of the left costophrenic angle (Fig. 6). These findings were consistent with previously published NS X-ray findings (11) and P3's specific medical history.

\section{DISCUSSION}

The technological improvement and increased use of lymphatic imaging over time have led to significant progress in our understanding of lymphatic phenotypes in NS and the lymphatic etiology of many NS sequelae. A comprehensive retrospective analysis of 52 patients from 21 lymphatic imaging publications, along with 2 of our own additional patients, supports the hypothesis that central lymphatic blockage due to lymph vessel/valvular dysplasia results in variably severe lymphatic reflux, which contributes significantly to the spectrum of congenital features, symptoms, and complications 

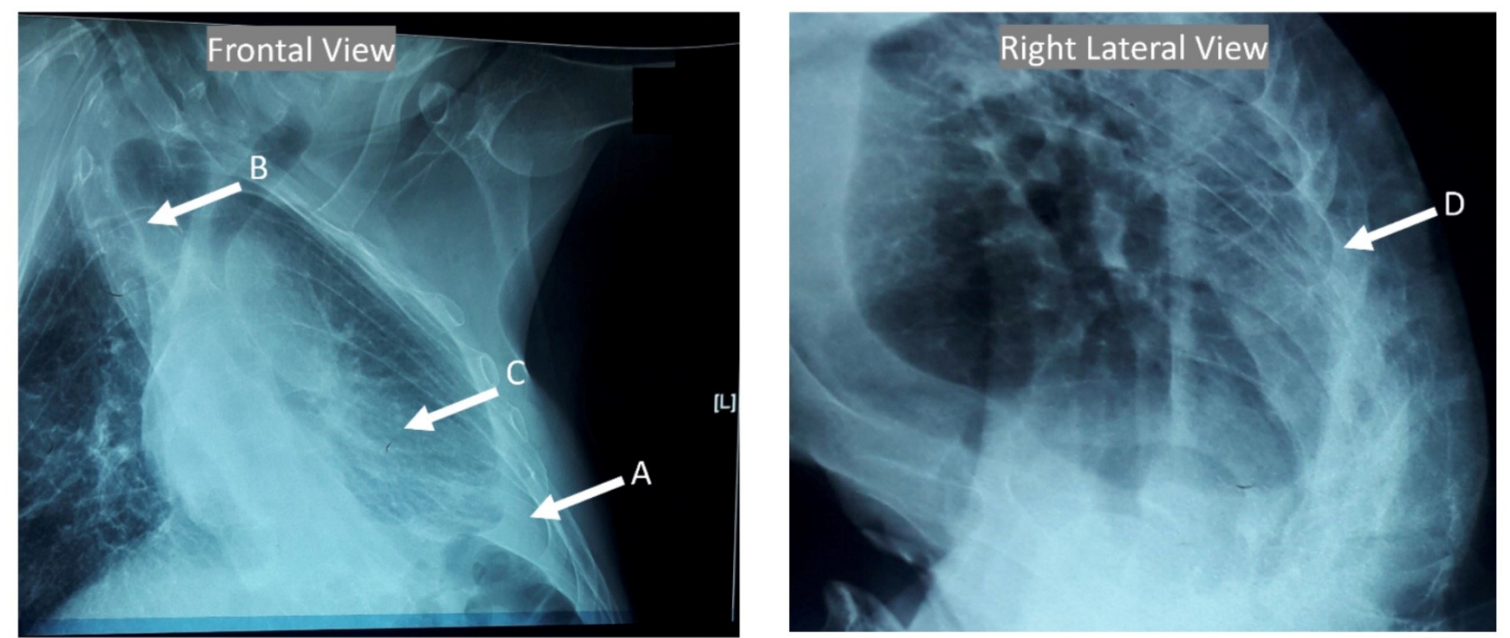

Fig. 6. Patient 3 CXR follow-up at age 29. A) Costophrenic angle blunted by fluid from persistent chylothorax (arrow A). Dextroscoliosis, a common musculoskeletal issue in NS patients (arrow B). Very fine reticulo-nodular pattern consistent with congestion of the pulmonary lymphatics (arrow C). Retrosternal density indicating disruption, congestion, or dilation of the internal mammary chain (helps drain the parietal pleural lymphatics) (arrow D).

associated with NS. Consequently, a NS diagnosis should promptly warrant anticipatory lymphatic imaging. Although ultrasound was not discussed due to the lack of lymphatic specificity, this imaging modality is useful for evaluating specific concerns such as renal tract abnormalities (12).

\section{Review of the Literature - Clinical Findings and Relevant Chronology}

All twenty-one studies observed anatomic abnormalities in the lymphatic vasculature of patients with NS. Additionally, several of these publications emphasized the near-ubiquitous presence of lymphatic dysplasia even without clinically apparent primary lymphedema. Fifteen studies specifically addressed or discussed abnormalities in lymphatic flow (Table 1.1 and 1.2). Of these, studies 16 [ $n=11(5)]$ and 19 [ $n=10(6)]$ contained the largest patient cohorts, on which the authors placed special emphasis for an association between the visualized obstruction of the thoracic duct and correspondingly poor clinical outcomes. Six studies did not make lymphatic flow observations (1318). These six studies used lymphatic imaging to emphasize other aspects of NS lymphatic pathogenesis such as specific genetic mutations or unique clinical manifestations.

Additional findings in the patients with NS that were reviewed included congenital heart defects (CHDs) such as pulmonic stenosis, that was present in 38/54 (70.4\%) patients. As a note, other studies with larger cohort sizes have observed CHDs in as many as $80 \%$ of patients with NS (19). Relevant clinical characteristics that led to a diagnosis of NS or to have lymphatic imaging performed included: cardiac defects $(n=38)$, peripheral lymphedema $(n=29)$, chylothorax $(n=22)$, lymphorrhea $(n=6)$, chylous ascites $(n=3)$, lympho-cutaneous fistula $(n=3)$, protein-losing enteropathy $(n=2)$, chylocolporrhea $(n=1)$, and chyluria $(n=1)$.

The imaging techniques used to evaluate the lymphatic system continue to evolve. Prior to 1984, C-LAG and CXR were the only reported methods for evaluation of the lymphatic system in patients with NS (Table 1.1 and 1.2). In 2015, direct injection of radiocontrast into the inguinal lymph nodes was 
TABLE 1.1

Chronology and Patient Representation of Various Imaging Modalities.

\begin{tabular}{|c|c|c|}
\hline Publication (year) & No. Patients & Imaging Studies \\
\hline Study 1 (1975) & 2 & C-LAG $(n=2)$ \\
\hline Study 2 (1976) & 1 & C-LAG $(n=1)$ \\
\hline Study 3 (1978) & 5 & C-LAG $(n=4)$ \\
\hline Study 4 (1980) & 2 & C-LAG $(n=2)$ \\
\hline Study 5 (1980) & 4 & $\operatorname{CXR}(n=4)$ \\
\hline Study 6 (1984) & 1 & $\operatorname{CXR}(n=1), \operatorname{LAS}(n=1)$ \\
\hline Study 7 (1997) & 1 & C-LAG $(n=1)$, CXR $(n=1)$ \\
\hline Study $8 *(1998)$ & 1 & C-LAG $(n=1), \operatorname{LAS}(n=1), \operatorname{MRL}(n=1)$ \\
\hline Study 9 (2003) & 1 & C-LAG $(n=1)$ \\
\hline Study 10 (2003) & 1 & $\operatorname{LAS}(n=1)$ \\
\hline Study 11 (2004) & 3 & $\operatorname{CXR}(n=3), \operatorname{LAS}(n=2), \operatorname{MRL}(n=1), \operatorname{CT}(n=3)$ \\
\hline Study 12 (2008) & 1 & $\operatorname{LAS}(n=1), \operatorname{MRL}(n=1)$ \\
\hline Study 13 (2011) & 1 & $\operatorname{LAS}(n=1), C T(n=1)$ \\
\hline Study 14 (2015) & 1 & $\operatorname{LAS}(n=1), \operatorname{MRI}(n=1), C T(n=1)$ \\
\hline Study 15 (2015) & 1 & C-LAG $(n=1), \operatorname{LAS}(n=1), C T(n=1)$ \\
\hline Study 16 (2016) & 11 & $\operatorname{LAS}(n=7)$ \\
\hline Study 17 (2018) & 1 & C-LAG $(n=1), C T(n=1), \operatorname{LAS}(n=1)$ \\
\hline Study 18 (2018) & 1 & $\operatorname{MRL}(n=1)$ \\
\hline Study 19 (2019) & 10 & C-LAG $(n=7), M R L(n=9)$, dc-MRL $(n=7)$ \\
\hline Study 20 (2020) & 2 & $\operatorname{LAS}(n=2), \operatorname{MRL}(n=2)$, I-LAG $(n=2)$ \\
\hline Study $21(2020)$ & 1 & dc-MRL $(n=1), I-L A G(n=1)$ \\
\hline $\begin{array}{c}\text { Cases 1-3* } \\
(2020)\end{array}$ & 2 & $\operatorname{CXR}(n=1), \operatorname{LAS}(n=3), \operatorname{MRL}(n=2), \operatorname{CT}(n=2), \operatorname{SPECT}(n=1)$ \\
\hline $\begin{array}{c}\text { Selected } \\
\text { Totals/Findings }\end{array}$ & 54 & $\begin{array}{l}\text { LAG }(n=24), \text { CXR }(n=10), \text { LAS }(n=22), \operatorname{MRL}(n=18), \\
\text { dc-MRL }(n=8), \text { CT }(n=9), \operatorname{SPECT}(n=1)\end{array}$ \\
\hline
\end{tabular}

Notes: When multiple imaging modalities are utilized in the associated study, " $n$ " indicates the number of patients. Six publications (studies 1, 2, 6, 13, 14, 18) did not specifically make lymphatic flow observations in NS.

Table Key (applicable to all tables):

$L A G=$ Conventional Oil-Lymphangiography; $C X R=$ Chest $X$-Ray; $C T=$ Computerized Tomography;

$L A S=$ Lymphangioscintigraphy; $M R L=$ Magnetic Resonance Lymphography;

$d c-M R L=$ dynamic contrast MRL; SPECT = Single-Photon Emission Computerized Tomography

*Patient 1's case was first published in study 8, and was not counted twice in the patient pool 


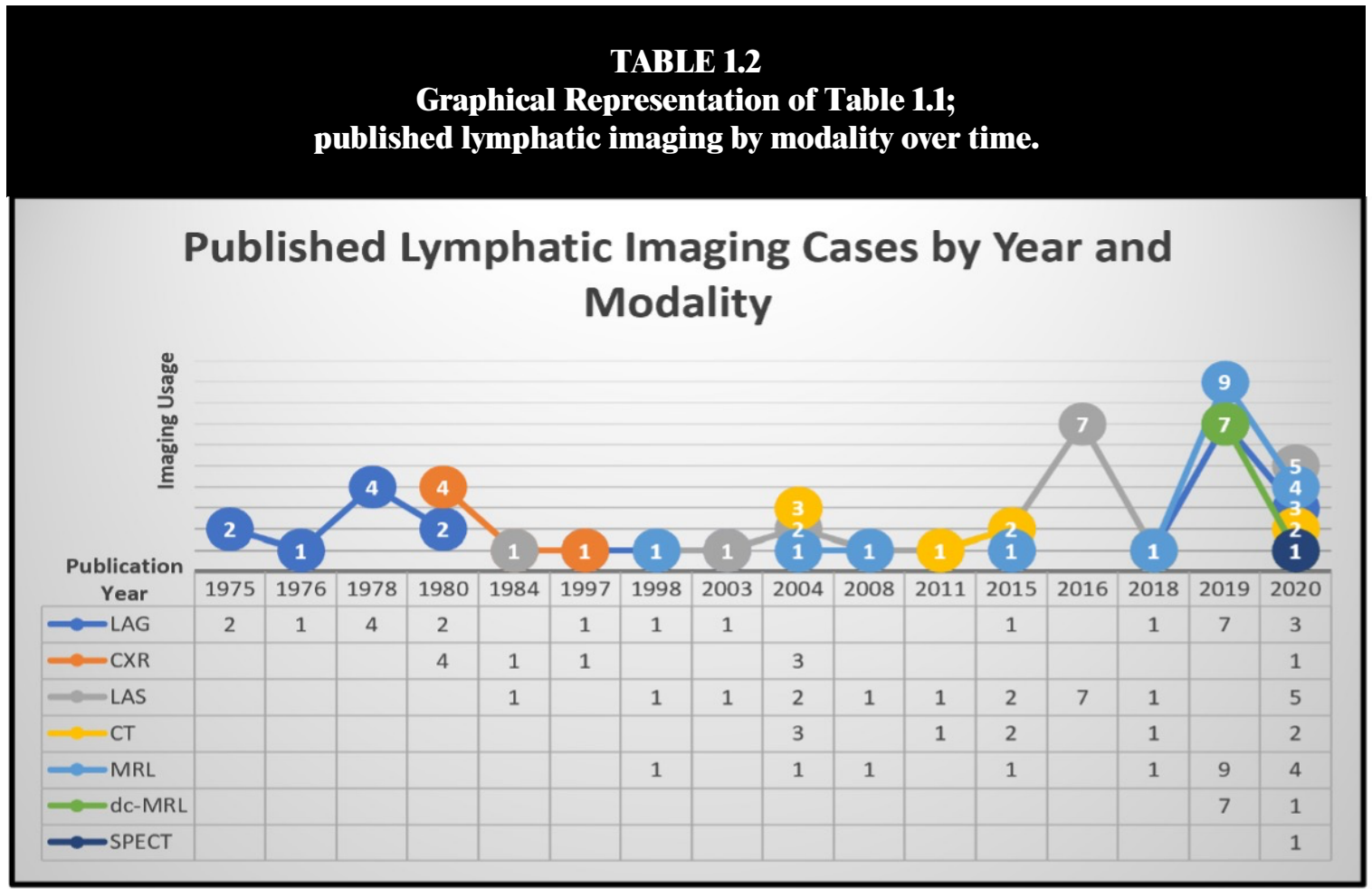

performed in a patient with $\mathrm{NS}$ - coined "intranodal LAG" (20) and presented by the authors as a less invasive and more versatile LAG imaging option for diagnostic and surgical purposes. The two most recently published studies utilized indocyanine green as a fluorescence-based contrast agent alternative to oil-based contrast for LAG imaging (21-22). LAS for NS patient evaluation was first introduced in 1984 (15) and has since been referred to by many as the "gold-standard" for dynamic evaluation of lymphatic function.

\section{Integrated Approach to Lymphatic Imaging in NS}

Based on this review of the literature, we have created a generalized approach to implement lymphatic imaging in the care of patients diagnosed with NS across the spectrum of disease presentation. Initial diagnosis of NS should promptly indicate a need for lymphatic imaging according to the clinical context surrounding the initial diagnosis. We review the uses of each imaging modality featured in the literature and integrate these findings into Table 2 and Fig. 7.

\section{Plain Chest Radiograph}

Hernandez et al (11) described unique and characteristic pulmonary patterns on CXR, which are postulated to be suggestive of risk for surgical complications in NS patients with pulmonary lymphangiectasia. Notably, when the internal mammary lymphatic vessels of the chest are dilated, they appear as an abnormal "retrosternal density" best appreciated on a lateral-view film. This key finding may preemptively signal higher risk of accidental damage during open heart surgery or may indicate blockage as a result of prior surgery. While the overall risk of postoperative pericardial and pleural effusions has been found to be similar in between NS patients compared to the general population 
TABLE 2

Summary Table of Lymphatic Imaging in NS Patient Care.

\begin{tabular}{|c|c|c|c|c|}
\hline Imaging Modality & Ideal Application(s) & Typical Findings & Advantages & Disadvantages \\
\hline $\begin{array}{l}\text { Lymphangiography } \\
\qquad(n=24)\end{array}$ & $\begin{array}{l}\text { Pre-operative planning; real- } \\
\text { time operative visualization; } \\
\text { embolization procedures }\end{array}$ & $\begin{array}{l}\text { Dysplasia, dilation, } \\
\text { collaterals, fistulae }\end{array}$ & $\begin{array}{l}\text { Detailed anatomical } \\
\text { representation of lympho- } \\
\text { vascular structure }\end{array}$ & $\begin{array}{l}\text { Risks of toxicity, embolism, } \\
\text { allergic reaction, and } \\
\text { infection; radio-exposure; } \\
\text { invasive; } 2 D\end{array}$ \\
\hline $\begin{array}{l}\text { Lymphangioscintigraphy } \\
\qquad(\mathrm{n}=22)\end{array}$ & $\begin{array}{c}\text { Planning of general long-term } \\
\text { management; pre/post- } \\
\text { operative lymphatic } \\
\text { evaluation }\end{array}$ & $\begin{array}{l}\text { Lymphstasis, reflux, delayed } \\
\text { Iymph flow rate }\end{array}$ & $\begin{array}{l}\text { Dynamic; less invasive; less } \\
\text { risk than LAG }\end{array}$ & $\begin{array}{l}\text { Low-resolution; radio- } \\
\text { exposure; time-consuming; } \\
\text { some patient discomfort; } 2 D\end{array}$ \\
\hline $\begin{array}{l}\text { Magnetic Resonance } \\
\text { Lymphography ( } n=18 \text { ) }\end{array}$ & $\begin{array}{l}\text { Evaluation of severe } \\
\text { widespread lymphatic } \\
\text { abnormalities }\end{array}$ & $\begin{array}{c}\text { Dysplasia, dilation, soft-tissue } \\
\text { effusions }\end{array}$ & $\begin{array}{l}\text { non-contrast; non-invasive; } \\
\text { no radio-exposure; 3D }\end{array}$ & $\begin{array}{l}\text { Expensive; time-consuming; } \\
\text { may require sedation }\end{array}$ \\
\hline Chest X-Ray $(n=10)$ & $\begin{array}{l}\text { Assessment of acute } \\
\text { respiratory symptoms; } \\
\text { screening for post-operative } \\
\text { complication risk }\end{array}$ & $\begin{array}{l}\text { Retrosternal densities, fine } \\
\text { reticulo-nodular pattern, } \\
\text { opaque fluid accumulation }\end{array}$ & Fast; inexpensive & $\begin{array}{l}\text { Interpretation-dependent; } \\
\text { non-specific; radio-exposure, } \\
2 D\end{array}$ \\
\hline CT Lymphography $(n=9)$ & $\begin{array}{l}\text { Apprciation of anatomic } \\
\text { relations in pre-operative } \\
\text { planning alongside C-LAG }\end{array}$ & $\begin{array}{l}\text { Dysplasia, leakage, contrast } \\
\text { accumulations }\end{array}$ & $\begin{array}{c}\text { Uses same radiocontrast as } \\
\text { LAG imaging; 3D }\end{array}$ & $\begin{array}{l}\text { Invasive; may require } \\
\text { sedation of young patients; } \\
\text { other risks similar to LAG }\end{array}$ \\
\hline $\begin{array}{l}\text { Dynamic-Contrast } \\
\text { Magnetic Resonance } \\
\text { Lymphography }(n=8)\end{array}$ & $\begin{array}{l}\text { Evaluation of severe } \\
\text { lymphatic abnormalities and } \\
\text { flow disturbances }\end{array}$ & $\begin{array}{l}\text { Dysplasia, dilation, missing } \\
\text { central lymphatic structures, } \\
\text { progression of lymphatic } \\
\text { reflux/effusion }\end{array}$ & $\begin{array}{l}\text { Extremely high resolution; } \\
\text { single-modality evaluation of } \\
\text { anatomy and physiology; 3D }\end{array}$ & $\begin{array}{l}\text { Invasive; may require } \\
\text { sedation of young patients }\end{array}$ \\
\hline $\mathrm{SPECT} / \mathrm{CT}(\mathrm{n}=1)$ & $\begin{array}{l}\text { Evaluation of severe } \\
\text { lymphatic abnormalities and } \\
\text { flow disturbances }\end{array}$ & $\begin{array}{l}\text { Lymphangiectasia, lymph } \\
\text { accumulation, reflux, dilation }\end{array}$ & $\begin{array}{l}\text { Combined-modality } \\
\text { evaluation of anatomy and } \\
\text { physiology; less invasive than } \\
\text { CTL or dc-MRL; 3D }\end{array}$ & $\begin{array}{l}\text { Radio-exposure; time } \\
\text { consuming (SPECT portion); } \\
\text { some patient discomfort }\end{array}$ \\
\hline
\end{tabular}

Notes: Imaging modalities are listed in descending order according to the value " $n$, which indicates the number of patients from the total patient pool $(n=54)$ that were imaged at least once. "Ideal applications" are generalized suggestions based on typical published findings as well as costs/benefits observed while managing NS patients.

with similar CHDs (23), the potential costs of routine CXR screening for at-risk NS patients may be far outweighed by the benefits associated with avoiding surgical complications.

\section{Lymphangiography and Computerized Tomography}

C-LAG is considered an invasive imaging method which involves surgical exploration and cannulation of lymphatic vessels in the hands or feet for injection of radiocontrast with subsequent visualization of the lymphatic vessels using X-ray. C-LAG identifies exact vascular structure, can be performed intraoperatively for real-time feedback, and can be performed alongside a CT for a comprehensive 3-D visualization of anatomic relationships with other organ systems. Intranodal LAG presents a more 

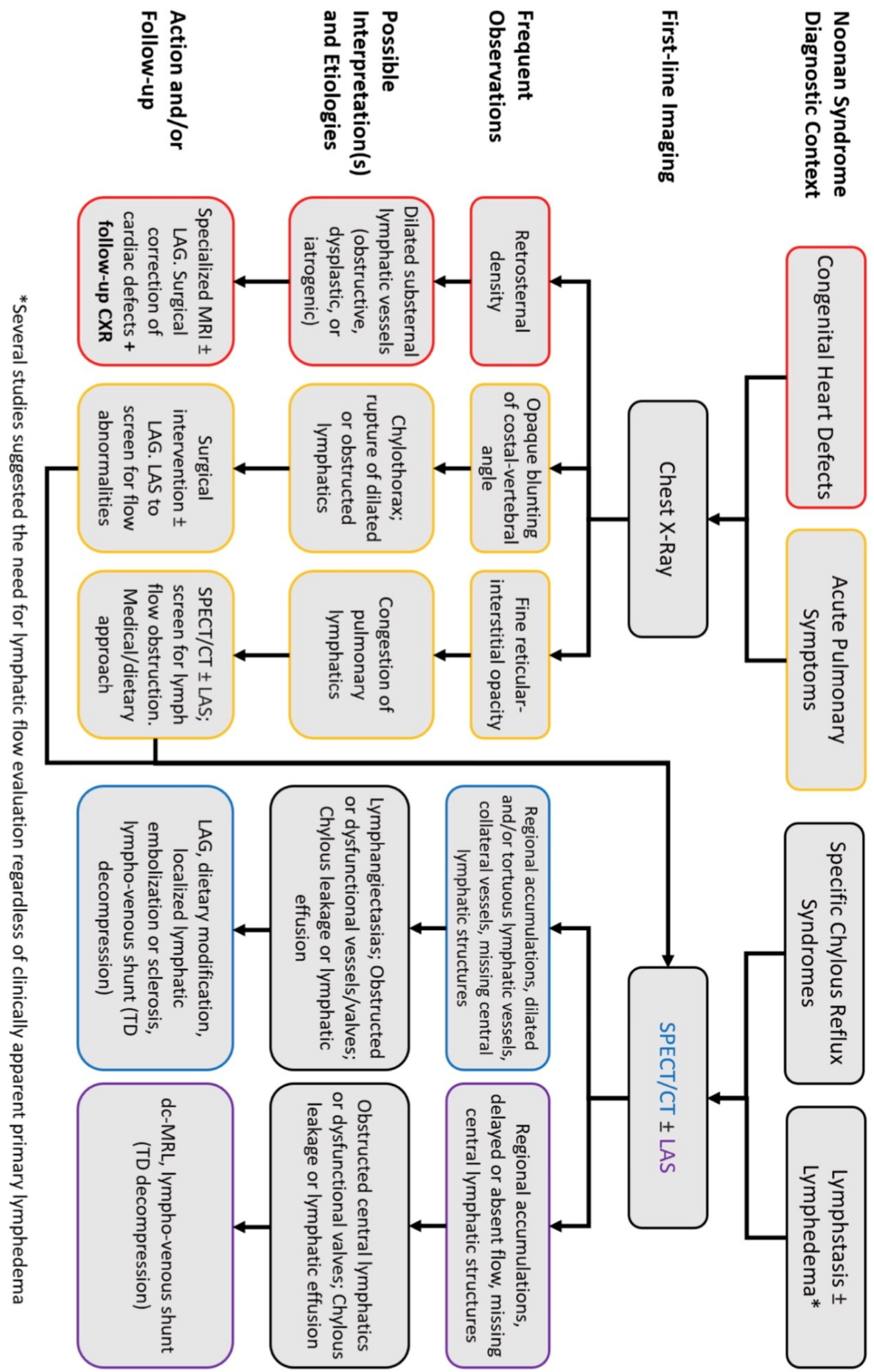

Fig. 7. Flow-chart illustration of a generalized NS case management plan, utilizing lymphatic imaging in various possible scenarios. This diagram is based on our comprehensive clinical analysis performed across the known literature. 
versatile LAG imaging option for diagnostic and surgical purposes (24). However, along with being invasive, C-LAG and CTL pose the most significant risk for imaging-related complications such as fat embolism, radiation exposure, and allergic reactions. I-LAG presents a safer imaging option than C-LAG due to decreased contrast toxicity and rapid hepatobiliary excretion (21-22). Indocyanine green may still exhibit allergenicity but overall has lower potential for toxicity and is rapidly excreted from the body via the hepatobiliary route. LAG remains an effective tool for surgical planning and execution given the modality's ability to map exact lymphatic architecture in real time.

\section{Magnetic Resonance Imaging}

Three-dimensional imaging of the lymphatic system in NS patients has been an area of rapidly developing interest along with a push for advanced imaging that can accurately depict lymphatic flow abnormalities. Heavily T2-weighted MRL can highlight slow-moving lymphatic fluid without the use of a contrast agent. On the other hand, T1-weighted dc-MRL uses contrast and captures images over specified time intervals to dramatically highlight the lymphatic vasculature of the patient and track abnormal fluid leakage into tissues as it appears. The limitations of this imaging method include the need for anesthesia in young patients and the invasiveness of inguinal lymph node cannulation (6).

\section{Planar Scintigraphy and Single-Photon Emission Computed Tomography/Computed Tomography/CT}

Procedurally, LAS can refer to planar imaging and/or SPECT/CT. Both methods begin with the requisite subdermal radiotracer injected into the peripheral lymphatics. The key difference between the two types of imaging is the scanning method; LAS-planar imaging collects a two-dimensional image only, while LAS-SPECT/CT can characterize both 3D anatomy and functionality of a patient's lymphatic system. The CT component of the SPECT/CT does expose the patient to a small amount of additional radiation. Typically, imaging begins with planar acquisition which allows for dynamic imaging over the area of injection and then also rapid imaging of the whole body. A single SPECT/CT acquisition requires approximately 15-20 minutes and typically covers one area of the body, for example, the adult chest. We typically begin with planar imaging in order to optimize the timing of the SPECT/CT acquisition of the body area of interest. The literature notes that preserved function of lymphatic flow and other normal SPECT findings may serve as a preliminary contraindication for invasive surgical treatment. Delayed visualization of the liver, absence of key structures, and abnormal accumulation of radiotracer can all indicate lymphatic flow abnormalities of varying severity.

\section{SUMMARY}

Table 2 contains an abbreviated representation of the pros, cons, and ideal usecase scenarios for the several lymphatic imaging modalities as discussed above. Table 3 provides additional reference for the general appearance of the lymphatic imaging modalities discussed. Comprehensive evaluation and lymphatic imaging of any given NS patient should follow a structured flow depending upon the individual disease presentation (Fig. 7).

Imaging of NS lymphatic abnormalities was vital in the assessment and care for each patient previously presented. Of the three cases described by us, P1 and P3 underwent surgical procedures guided by several lymphatic imaging modalities, achieved long term quality-of-life improvements, and experienced some regression of their initial lymphatic symptoms. For P2, our novel use of SPECT/CT hybrid imaging represents 


\section{TABLE 3}

Select Examples of Imaging Studies Reviewed Here.

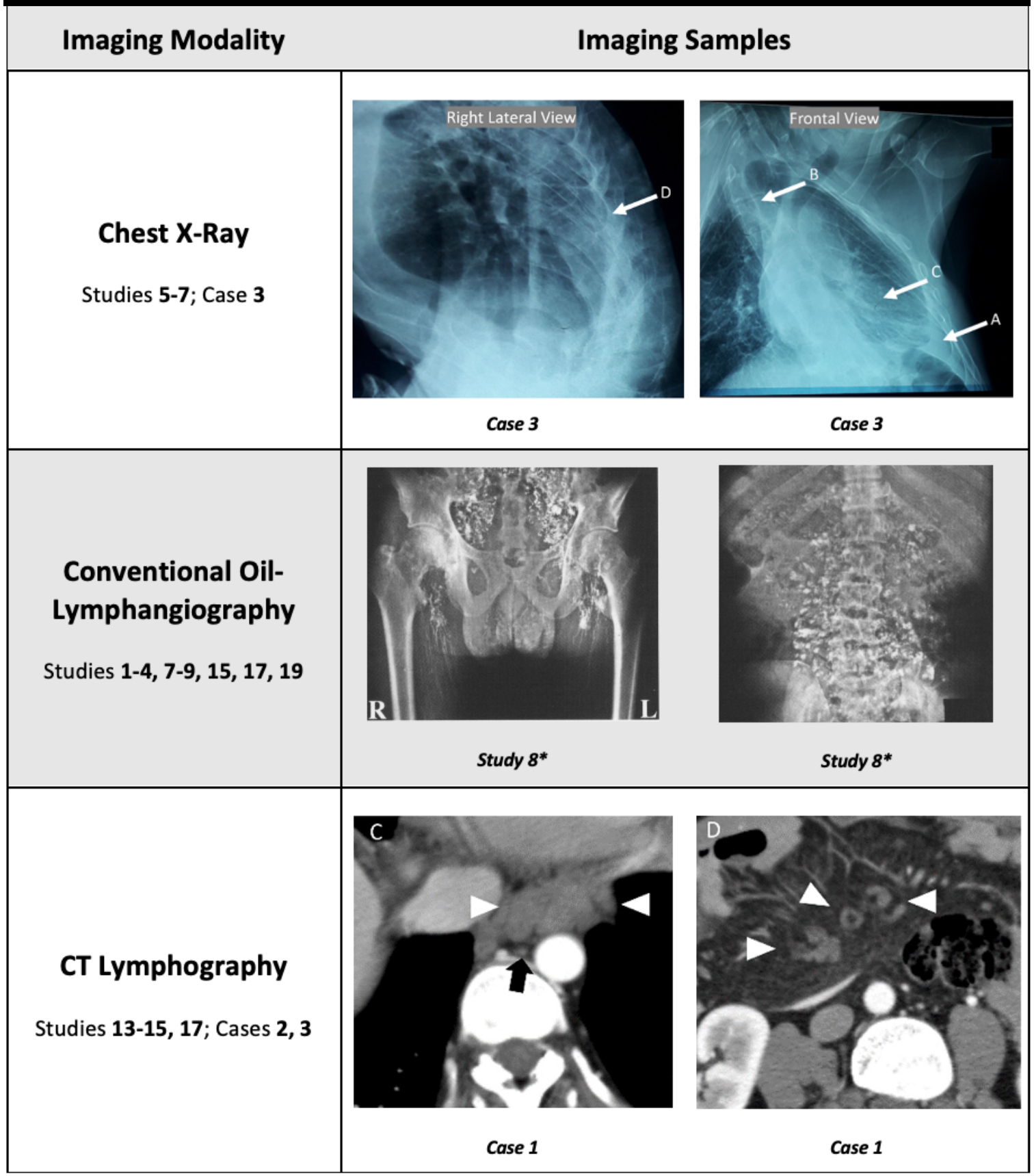


TABLE 3 (continued)

Select Examples of Imaging Studies Reviewed Here.

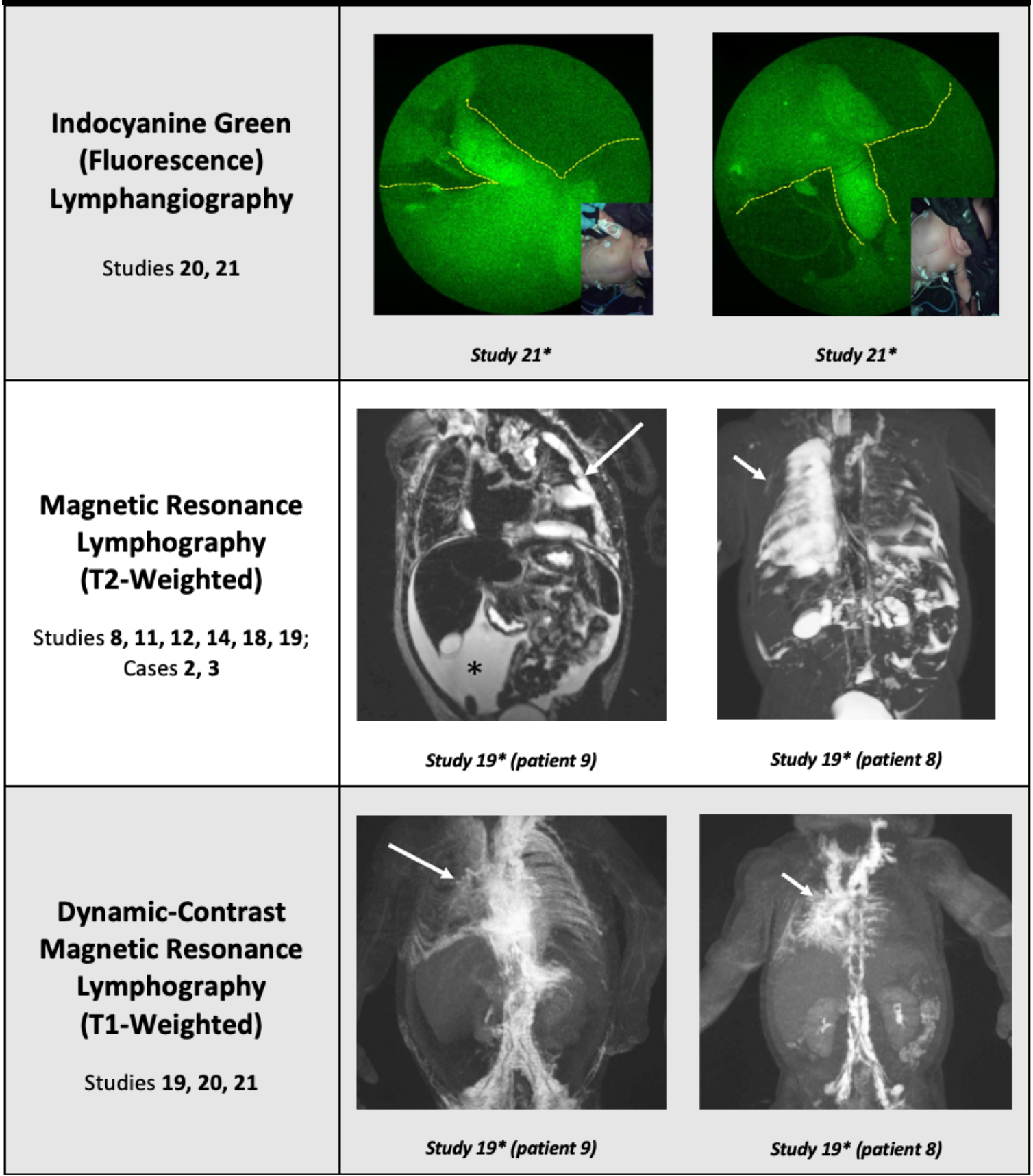




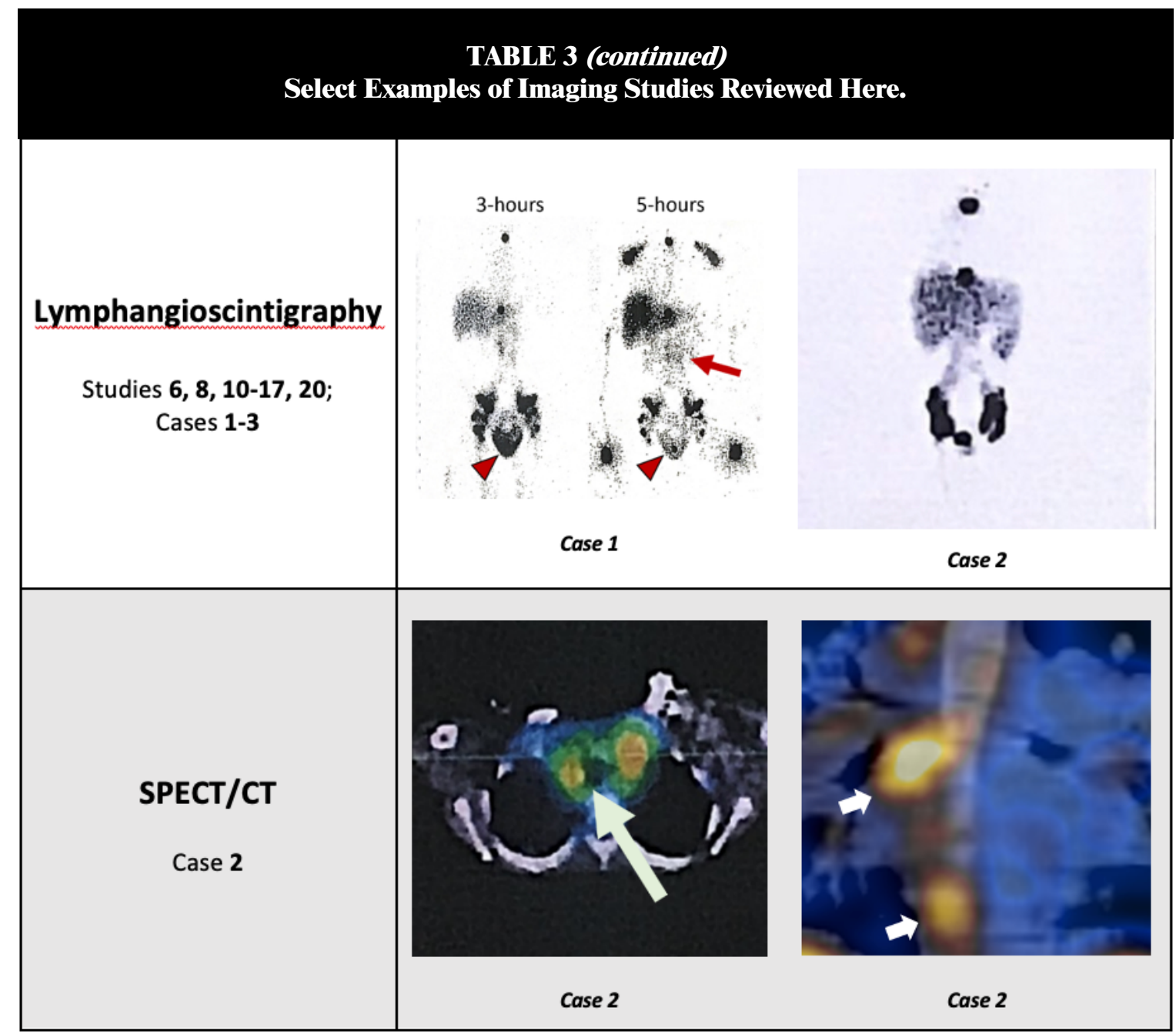

*Open-Access: permissions for image re-publication contingent on proper citation and author credit; see "References/Acknowledgements" and "Permissions"

significant technical progress in nuclear medicine imaging for NS patients (Fig. 3, 4). This technique enabled an evaluation of lymphatic function/structure in greater detail while simultaneously enhancing patient comfort and minimizing the risks of contrastrelated complications. Utilization of this method may help reduce the number of imaging studies necessary to evaluate lymphatic manifestations in NS patients moving forward.

\section{CONCLUSIONS}

The prioritization and use of different lymphatic imaging modalities for early evaluation and diagnosis of patients with NS should be assessed on an individual basis and the findings should be central to the creation of a comprehensive medical or surgical treatment plan for these patients. As with all medical imaging, costs and benefits of available imaging options and the urgency of 
immediate care must be considered when managing patients with NS. Skilled clinical observations and appropriate lymphatic imaging should be performed to characterize lymphatic phenotypes before effective and appropriate interventions can be individualized.

\section{CONFLICT OF INTEREST AND DISCLOSURE}

The authors declare no competing financial interests exist.

\section{ACKNOWLEDGMENTS}

The present study was supported in part by funds from the NIH National Heart, Lung and Blood Institute (R25HL108837 to TC and T35HL007479 to CV) and intramural Translational Imaging Program Projects Stimulus Funds to MHW.

\section{REFERENCES}

1. Deng, Y, D Atri, A Eichmann, M Simons: Endothelial ERK signaling controls lymphatic fate specification. J Clin Invest. 2013 Mar;123(3):1202-15. doi: 10.1172/JCI63034. Epub 2013 Feb 8. PMID: 23391722; PMCID: PMC3582116.

2. Noonan JA. Hypertelorism with Turner phenotype. A new syndrome with associated congenital heart disease. Am J Dis Child. 1968 Oct;116(4):373-80. doi: 10.1001/archpedi.1968.02100020377005. PMID: 4386970.

3. Minkin W, Frank SB, Wolman SR, Cohen HJ. Lymphedema in Noonan's syndrome. Int J Dermatol. 1974 Jul-Aug;13(3):179-83. doi: 10.1111/j.1365-4362.1974.tb01788.x. PMID: 4429607.

4. (Study 14) Morcaldi, G, T Bellini, C Rossi, et al: Lymphodysplasia and kras mutation: A case report and literature review. Lymphology 48 (2015) 121-127.

5. (Study 16) Joyce, S, K Gordon, G Brice, et al: The lymphatic phenotype in Noonan and Cardiofaciocutaneous syndrome. Eur. J. Hum. Genet. 24 (2016), 690-696.
6. (Study 19) Biko, DM, B Reisen, HJ Otero, et al: Imaging of central lymphatic abnormalities in Noonan syndrome. Pediatr. Radiol 49 (2019), 586-592.

7. Gaudineau, A, B Doray, E Schaefer, et al: Postnatal phenotype according to prenatal ultrasound features of Noonan syndrome: A retrospective study of 28 cases. Prenat. Diagn. 33 (2013), 238-241.

8. (Study 8) Howarth D, Gloviczki P. Lymphoscintigraphy and lymphangiography of lymphangiectasia. J. Nucl. Med. 39 (1998), 1635-1638.

9. Seo, Y, C Mari, BH Hasegawa: Technological development and advances in single-photon emission computed tomography/computed tomography. Semin. Nucl. Med. 38 (2008),177-198.

10. Lepri, F, A De Luca, L Stella, et al: SOS1 mutations in Noonan syndrome: Molecular spectrum, structural insights on pathogenic effects, and genotypephenotype correlations. Hum. Mutat. 32 (2011), 760-762.

11. (Study 5) Hernandez, RJ, AM Stern, A Rosenthal: Pulmonary lymphangiectasis in Noonan syndrome. AJR Am J Roentgenol. 134 (1980), 75-80.

12. George, CD, MA Patton, M el Sawi, M Sharland, EJ Adam EJ: Abdominal ultrasound in Noonan syndrome: a study of 44 patients. Pediatr. Radiol. 23 (1993), 316-318.

13. (Study 1) Baltaxe, HA, JG Lee, KH Ehlers, MA Engle: Pulmonary lymphangiectasia demonstrated by lymphangiography in 2 patients with Noonan's syndrome. Radiology 115 (1975), 149-153.

14. (Study 2) Herzog, DB, R Logan, JB Kooistra: The Noonan syndrome with intestinal lymphangiectasia. J. Pediatr. 88 (1976), 270-272.

15. (Study 6) Sty, JR, JP Thomas Jr, MH Wolff, SB Litwin: Lymphoscintigraphy. Pulmonary lymphangiectasia. Clin. Nucl. Med. 9 (1984), 716.

16. (Study 13) Goli, S, V Kandimalla, O Khan: A case of Noonan's syndrome with anomalous lymphorenal communication. JRSM Short Rep. 18 (2011), 33.

17. (Study 11) Bellini, C, M Mazzella, $C$ Campisi, et al: Multimodal imaging in the congenital pulmonary lymphangiectasia- 
congenital chylothorax-hydrops fetalis continuum. Lymphology 37 (2004), 22-30.

18. (Study 18) Bobot, M, M Coen, C Simon, et al: DRESS syndrome with thrombotic microangiopathy revealing a Noonan syndrome: Case report. Medicine (Baltimore) 97 (2018), e0297.

19. Prendiville, TW, K Gauvreau, E TworogDube, et al: Cardiovascular disease in Noonan syndrome. Arch. Dis. Child. 99 (2014), 629-634.

20. (Study 15) Matsumoto, T, T Kudo, J Endo, et al: Transnodal lymphangiography and post-CT for protein-losing enteropathy in Noonan syndrome. Minim. Invasive Ther. Allied Technol. 24 (2015), 246-249.

21. (Study 21) Pham, KT, D Balaguru, VS Tammisetti, et al: Multimodality lymphatic imaging of postoperative chylothorax in an infant with Noonan syndrome: A case report. Eur. J. Med. Res. 25 (2020), 55.

22. (Study 20) Liu, NF, MZ Gao: Lymphatic system malformations in Noonan syndrome: Two case reports and imaging analysis. Lymphology 53 (2020), 76-80.

23. Landis BJ, DS Cooper, RN Hinton: CHD associated with syndromic diagnoses: perioperative risk factors and early outcomes. Cardiol. Young. 26 (2016), 30-52.

24. (Study 17) Yamamoto, M, H Kondo, F Shigeru, et al: Intranodal lymphatic embolization for chylocolporrhea caused by chylous reflux syndrome in noonan syndrome. J. Vasc. Interv Radiol. 30 (2019), 769-772.

\section{Additional Review Articles}

Study 3) Lanning P, S Similä, I Suramo, T Paavilainen: Lymphatic abnormalities in Noonan's syndrome. Pediatr. Radiol. 19 (1978), 106-109.

Study 4) Hoeffel, JC, P Juncker, J Remy: Lymphatic vessels dysplasia in Noonan's syndrome. Am. J. Roentgenol. 134 (1980), 399401.

Study 7) Bloomfield FH, W Hadden, TR Gunn: Lymphatic dysplasia in a neonate with Noonan's syndrome. Pediatr. Radiol. 27 (1997), 321-323.

Study 9) Ho, WL, JK Wang, YW Li:

Radiological features of late-onset lymphoedema in Noonan's syndrome. Pediatr. Radiol. 33 (2003), 200-202.

Study 10) Ogata, T, S Sato, Y Hasegawa, K Kosaki: Lymphstasis in a boy with Noonan syndrome: Implication for the development of skeletal features. Endocr J. 50 (2003), 319-324.

\author{
Marlys H. Witte, MD \\ Professor of Surgery, Neurosurgery, \& \\ Pediatrics \\ University of Arizona College of Medicine \\ 1501 N. Campbell Avenue, Room 4402J \\ Tucson, AZ 85724-5200 USA \\ Phone: (520) 626-6118 \\ E-mail: lymph@surgery.arizona.edu
}

